\title{
LINFOMAS ORBITARIOS. PRESENTACIÓN DE NUEVE CASOS
}

\section{ORBITAL LYMPHOMAS. PRESENTATION OF NINE CASES}

\author{
REY-PORCA $\mathrm{C}^{1}$, PÉREZ-ENCINAS M${ }^{2}$, GONZALEZ $\mathrm{F}^{3}$
}

\begin{abstract}
RESUMEN
Objetivo: Describir nueve casos de linfomas orbitarios.

Métodos: Revisión de historias clínicas de nueve pacientes diagnosticados de linfoma orbitario y consulta de la bibliografía relacionada con esta patología.

Resultados: Se presenta una serie compuesta por cinco mujeres y cuatro varones con linfoma en la región orbitaria. En nuestros casos, la mayoría de los pacientes presentaron linfoma extraorbitario concurrente en el momento en el que el proceso orbitario fue detectado por primera vez (siete de los nueve pacientes). Tres de los pacientes presentaron linfoma MALT, dos linfomas foliculares, dos linfomas no Hodgkin de células B grandes, un linfoma de células B de bajo grado y un linfoma de células del manto. Ocho pacientes se mantienen vivos y uno ha fallecido a consecuencia de su linfoma en el momento de escribir este artículo.

Conclusiones: Se ha observado un incremento de la incidencia de los linfomas no Hodgkin orbitarios durante las últimas tres décadas. El tipo más común en la región orbitaria es el linfoma MALT. La forma
\end{abstract}

\section{ABSTRACT}

Purpose: To report nine cases of orbital lymphomas. Methods: We reviewed the clinical records of nine patients diagnosed with orbital lymphoma and performed a literature search related to this condition. Results: We present a series of five women and four males with orbital lymphoma involving the orbital region. In our cases, most patients presented concurrent extraorbital lymphoma when the orbital disease was first noticed (seven out of nine patients). We found three MALT lymphomas, two follicular lymphomas, two non-Hodgkin large B cell lymphomas, one low grade B cell lymphoma, and one mantle cell lymphoma. Eight patients were alive and one had died as a consequence of his lymphoma at the time this report was written.

Conclusions: An increase in the incidence of nonHodgkin orbital lymphomas has been observed over the last three decades. The most common type in the orbital region is the MALT lymphoma. The clinical features observed in our series are similar to those reported in the literature. Since lymphomas are the most frequent malignant tumours in the orbit, usually with extraorbital involvement, and

\footnotetext{
Recibido: 5/2/07. Aceptado: 17/1/08.

1 Licenciado en Medicina. Servicio de Oftalmología del Complejo Hospitalario Universitario de Santiago de Compostela. España.

2 Doctor en Medicina. Servicio de Hematología. Complejo Hospitalario Universitario de Santiago de Compostela. España.

3 Doctor en Medicina. Servicio de Oftalmología del Complejo Hospitalario Universitario de Santiago de Compostela. España; y Departamento de Fisiología. Facultad de Medicina de la Universidad de Santiago de Compostela. España.

Este trabajo ha sido realizado gracias a los fondos del proyecto BFU2004-01839 del Ministerio de Educación y Ciencia y FEDER.
}

Correspondencia:

Francisco González

Departamento de Fisiología. Facultad de Medicina

Universidad de Santiago de Compostela

15782 Santiago de Compostela

España

E-mail: francisco.gonzalez@usc.es 
de presentación clínica que encontramos en nuestra serie es similar a la que se presenta en otros estudios. Dado que los linfomas son los tumores malignos más frecuentes en la órbita, habitualmente tienen también localización extraorbitaria, y pueden ser tratados con éxito en muchos casos, es importante que el oftalmólogo tenga presente esta patología.

Palabras clave: Órbita, linfoma, linfoma de anejos oculares, tumores orbitarios, linfomas no Hodgkin orbitarios. can be successfully treated in many cases, it is important for the ophthalmologist to be aware of this condition (Arch Soc Esp Oftalmol 2008; 83: 95-104).

Key words: Orbit, lymphomas, ocular adnexal lymphoma, orbital tumours, non-Hodgkin orbital lymphomas.

\section{INTRODUCCIÓN}

Los linfomas son los tumores malignos más frecuentes de la órbita. Según recientes publicaciones suponen el $11 \%$ de todos los tumores orbitarios (1) y el $55 \%$ de los malignos (2). Esta alta prevalencia y la posibilidad de que tengan un tratamiento efectivo para su curación, hace que este tipo de neoplasias sea importante para el oftalmólogo. En este artículo presentamos nueve casos de linfomas orbitarios junto con una revisión bibliográfica relacionada con esta patología.

\section{SUJETOS, MATERIAL Y MÉTODOS.}

Se presentan nueve casos de linfomas orbitarios, cinco mujeres y cuatro varones, que han sido atendidos en el Servicio de Oftalmología de nuestro centro desde julio de 2002 hasta septiembre de 2006. La información bibliográfica se obtuvo mediante una búsqueda en la literatura médica utilizando fundamentalmente la base de datos PubMed (National Library of Medicine, EE.UU.) para analizar y contrastar nuestros hallazgos. A continuación se describen las características clínicas y evolución de los casos de esta serie.

\section{RESULTADOS}

\section{Caso 1}

Mujer de 69 años de edad que refería ptosis palpebral derecha desde septiembre de 2002 sin otra sintomatología (fig. 1A). Acudió a consulta de medicina interna y de neurología a los 15-20 días de su presentación. Una TAC realizada un mes más tarde mostró una lesión de aproximadamente 1,5 x $1,8 \mathrm{~cm}$ de tamaño en la parte central del párpado superior derecho (fig. 1B), y es remitida a nuestro Servicio de Oftalmología. La RMN (fig. 1C) muestra una lesión del mismo tamaño localizada por detrás del septum y encima del músculo elevador del párpado. Al acceder quirúrgicamente a la lesión se observó que infiltraba el tendón del músculo elevador y se extirpó. El diagnóstico anatomopatológico fue de linfoma MALT. La paciente fue remitida al Servicio de Hematología, donde se realizó el estudio de extensión, objetivándose localización única en la órbita derecha. Se indicó tratamiento con radioterapia y actualmente la paciente se encuentra estable sin recidivas.

\section{Caso 2}

Mujer de 60 años de edad que ingresó en el servicio de Medicina Interna en noviembre de 2004 para estudio de tumefacción del párpado superior derecho y poliadenopatías cervicales que en los últimos siete meses habían aumentado de tamaño, apareciendo progresivamente astenia, anorexia, pérdida de peso y sudoración nocturna. Mediante una biopsia medular y ganglionar se diagnosticó un linfoma no Hodgkin de células del manto, estadío IVB, recibiendo quimioterapia, con lo que se produjo remisión parcial. En agosto de 2005 se realizó una TAC, que puso de relieve una recidiva de la tumoración palpebral (fig. 2), por lo que fue remitida a nuestro Servicio de Oftalmología en septiembre de 2005. Dado el crecimiento rápido de la tumoración, se inició otro ciclo de quimioterapia e inmunoterapia con la que se obtuvo una rápida respues- 

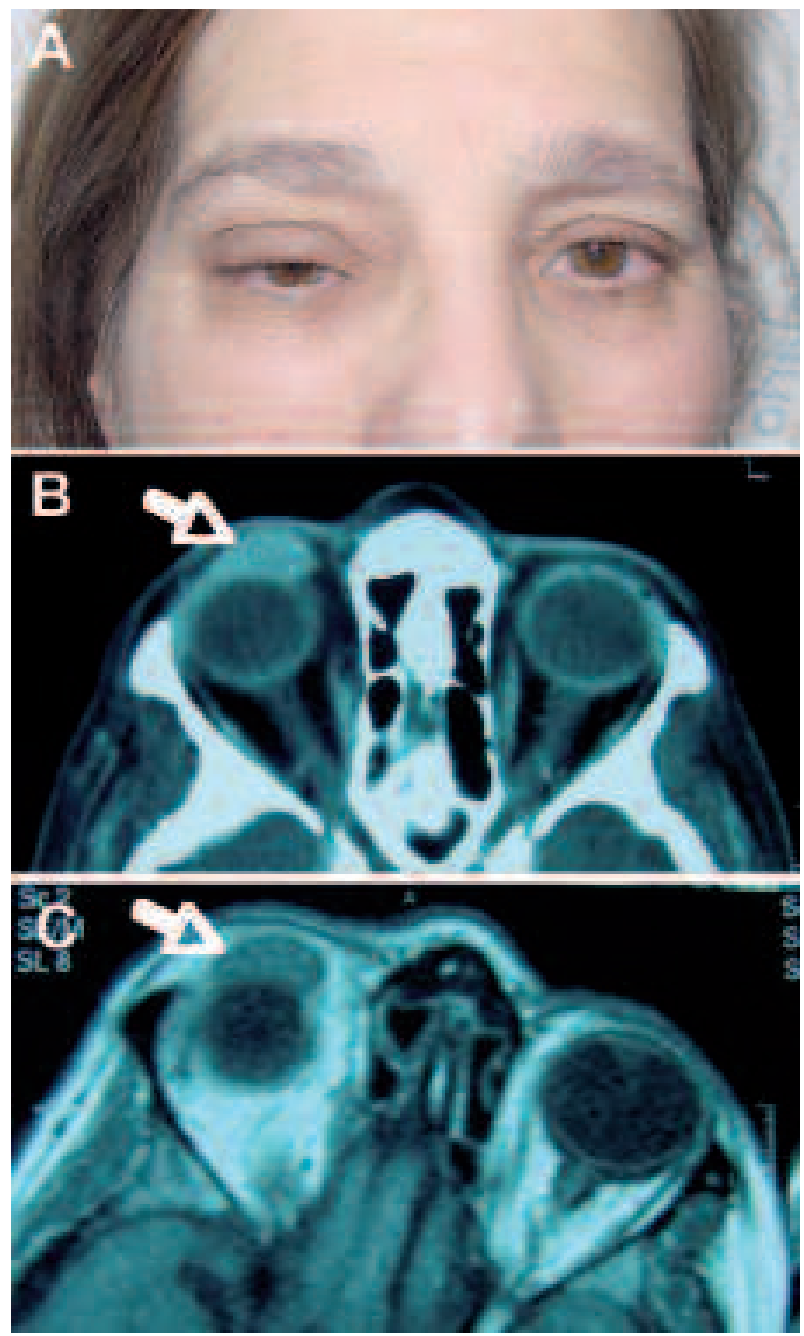

Fig. 1: Caso 1. La paciente presenta una ptosis de OD secundaria a una masa palpebral (A). En el TAC orbitario $(B)$ con contraste intravenoso y ventana de partes blandas se observa una masa a nivel del párpado superior derecho, adyacente al globo ocular (flecha). RMN orbitaria $(C)$ potenciada en T1 y con contraste paramagnético que muestra la localización del tumor (flecha).

ta. En marzo de 2006 presentó una nueva recidiva palpebral que volvió a ser tratada con quimioterapia con discreta respuesta. Actualmente la paciente continúa con su proceso en progresión.

\section{Caso 3}

Varón de 50 años de edad que fue atendido en otro Centro por presentar exoftalmo discreto en el
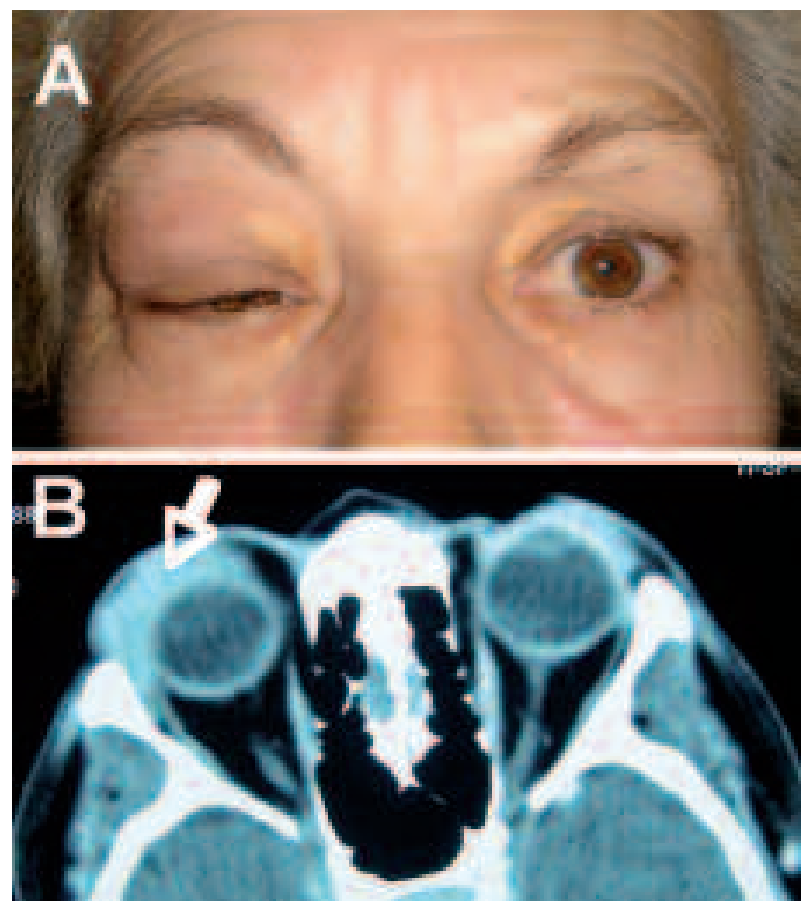

Fig. 2: Caso 2. En la imagen superior (A) se aprecia una tumefacción palpebral del ojo derecho producida por la tumoración que se observa en la TAC con contraste intravenoso de la imagen inferior $(B)$ indicado por la flecha.

ojo izquierdo de un mes de evolución (fig. 3A). Como antecedentes de interés refería un derrame pleural tuberculoso en el año 2000. La exploración oftalmológica constató masas infraorbitarias y supraorbitarias que se desplazaban a la palpación, con un exoftalmo de aproximadamente $3 \mathrm{~mm}$ con respecto al otro ojo. El resto de la exploración oftalmológica fue normal. Una TAC previa realizada en junio del 2002 mostró una masa retroocular izquierda que se cataloga como posible pseudotumor inflamatorio. En una RMN orbitaria realizada en junio de 2002 (fig. 3B), se planteó como primera posibilidad diagnóstica un linfoma orbitario o una metástasis, por lo que es remitido a nuestro Servicio de Oftalmología en julio de 2002, en donde se realizó una PAAF que fue compatible con un linfoma no Hodgkin. Una biopsia posterior confirmó el diagnostico de linfoma B de bajo grado positivo para CD20 y Bcl-2 (sin poder especificar el tipo). El estudio de extensión demostró la ausencia de otros focos tumorales y se indicó tratamiento radioterápico. El paciente continúa en remisión completa. 

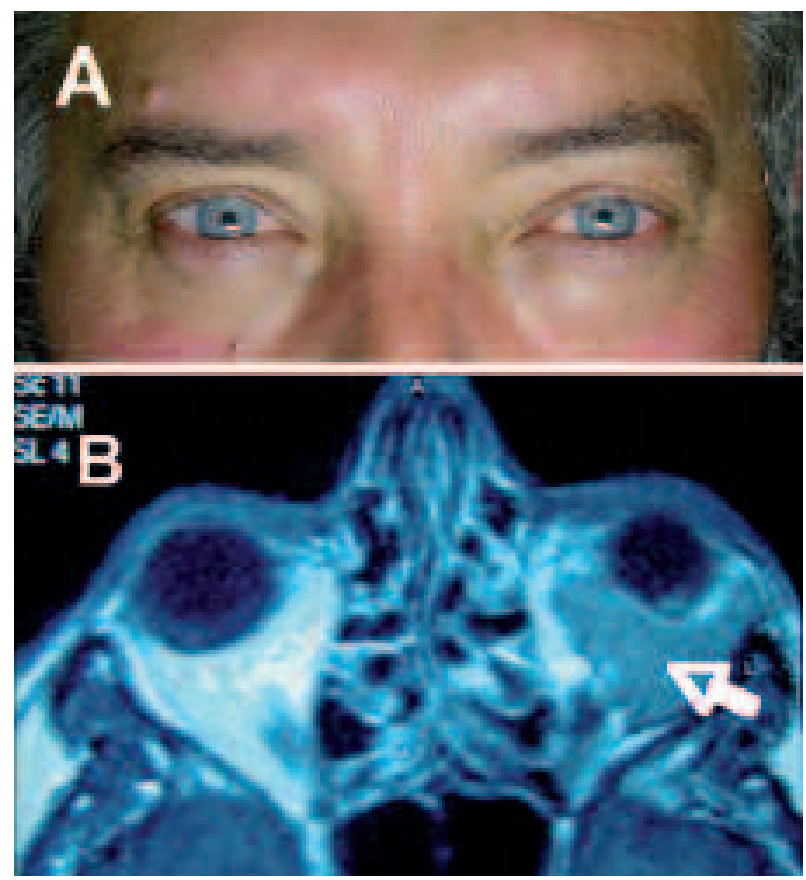

Fig. 3: Caso 3. El paciente presenta un ligero exoftalmo izquierdo (A). La imagen de RMN (B) potenciada en T1 con contraste muestra una masa infraorbitaria izquierda retroocular.

\section{Caso 4}

Varón de 53 años de edad que acudió a nuestra Consulta de Oftalmología en abril de 2004 por presentar una tumoración blanda y reductible de apro-

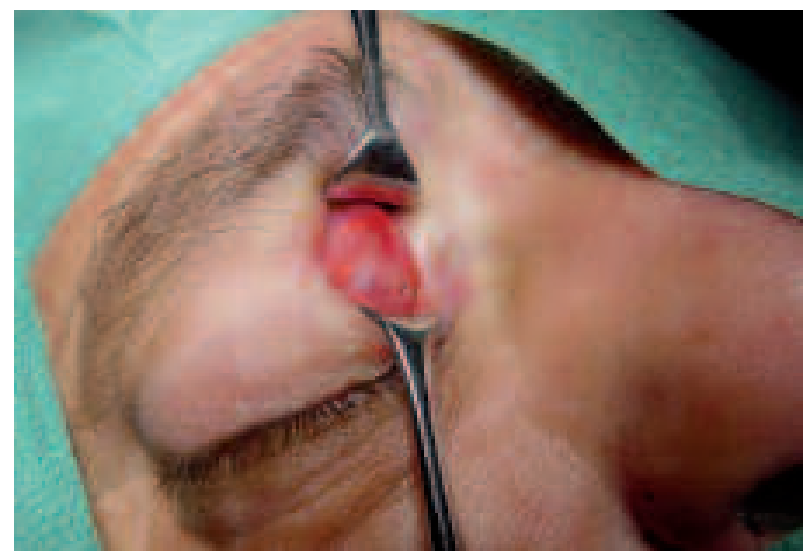

Fig. 4: Caso 4. En este caso, el linfoma se presentó como una pequeña tumoración en la parte interna del párpado superior. Se procedió a su extirpación sin conocer previamente el diagnóstico. ximadamente $1 \mathrm{~cm}$ de diámetro, localizada en la porción medial del párpado superior derecho (fig. 4). El enfermo no presentaba antecedentes de interés. Un mes más tarde se extirpó la tumoración, de consistencia semiblanda, con pseudocápsula y polilobulada, que se extendía por la pared interna de la órbita hasta el ápex sin afectar hueso. El diagnóstico anatomopatológico fue de linfoma MALT. Se remitió a hematología, donde se detectó una posible afectación cervical derecha por PET y se instauró tratamiento con radioterapia en órbita y cuello. El paciente continúa en remisión en la actualidad.

\section{Caso 5}

Varón de 71 años de edad diagnosticado en octubre de 2001 de linfoma B de células grandes de nasofaringe con afectación del oído medio, seno maxilar derecho, adenopatías cervicales derechas y posible afectación pleural y de médula ósea por citometría de flujo. Se inició tratamiento quimioterápico y radioterápico locorregional con respuesta completa. En marzo de 2004 comenzó con edema del párpado inferior derecho, que dos meses después ya dificultaba la apertura palpebral (fig. 5A). No presentaba ninguna otra sintomatología relevante. Una TAC de órbita realizada en mayo de 2004 objetivó una masa de densidad de tejidos blandos en la parte ínfero-interna de la órbita derecha (fig. 5B) que mediante una PAAF confirmó la sospecha de recidiva del linfoma. El estudio de extensión mostró infiltración en médula ósea. Recibió de nuevo quimioterapia sistémica y radioterapia sobre órbita derecha. El paciente presentó una nueva recidiva en región facial (una adenopatía submentoniana derecha) dos años después, por lo que ha recibido tratamiento radioterápico, encontrándose asintomático en la actualidad.

\section{Caso 6}

Mujer de 55 años de edad que acudió a nuestro Servicio de Oftalmología en enero de 2002 por presentar una tumoración en la fosa lagrimal izquierda que producía ptosis palpebral y desplazamiento inferior del globo (fig. 6A). Una TAC orbitaria confirmó la existencia de una masa en la fosa lagrimal izquierda, que fue extirpada en febrero de 2002. El diagnóstico anatomopatológico fue de linfoma B de 

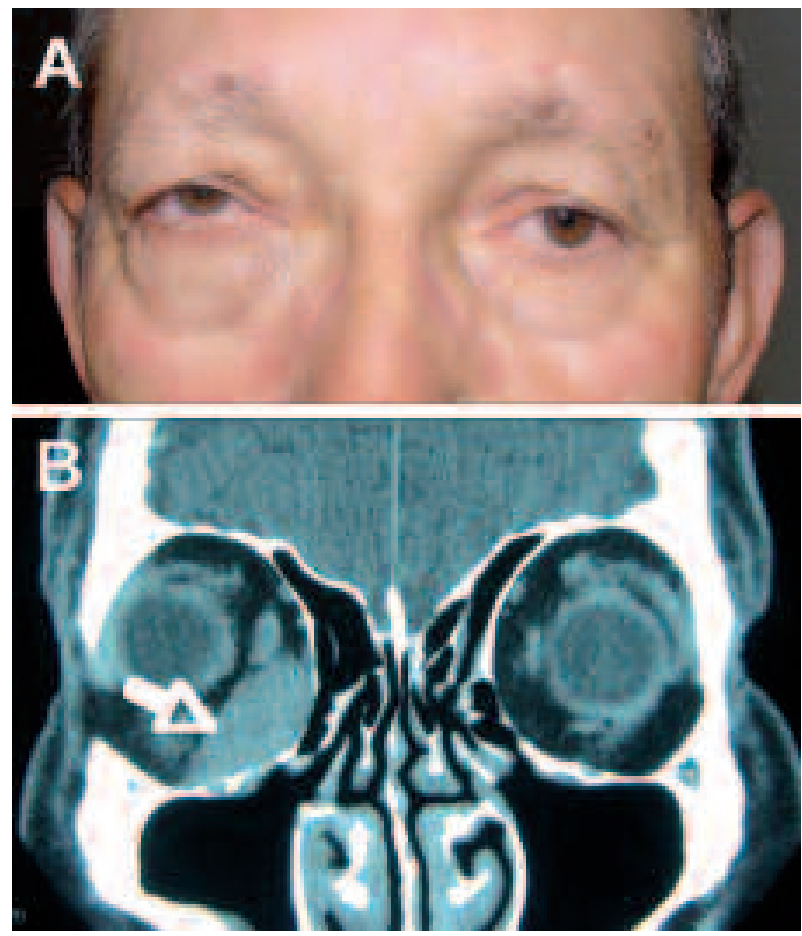

Fig. 5: Caso 5. Se observa la tumefacción del párpado inferior derecho (A). El TAC craneal sin contraste (B) muestra una masa en la zona ínfero-interna de la órbita derecha (flecha).

bajo grado. La paciente no acudió a nuevas consultas hasta septiembre de 2003, fecha en la que presentaba una tumoración preauricular izquierda (fig. 6B), que al ser biopsiada confirmó el diagnóstico de linfoma folicular. En un estudio de extensión se determinó un estadio IV. Se inició tratamiento quimioterápico y la paciente actualmente está asintomática.

\section{Caso 7}

Mujer de 64 años de edad que fue diagnosticada en mayo de 2000 de linfoma MALT pulmonar mediante biopsia transbronquial con estudio de extensión mostrando mínima infiltración en médula ósea por citometría de flujo. Recibió tratamiento con quimioterapia con respuesta parcial. En octubre de 2002 se detectó progresión a nivel pulmonar e infiltración de médula ósea, pautándose un nuevo tratamiento quimioterápico e inmunoterápico, obteniéndose una respuesta parcial. En febrero de 2006, cuando se realizó un nuevo estudio de extensión, se
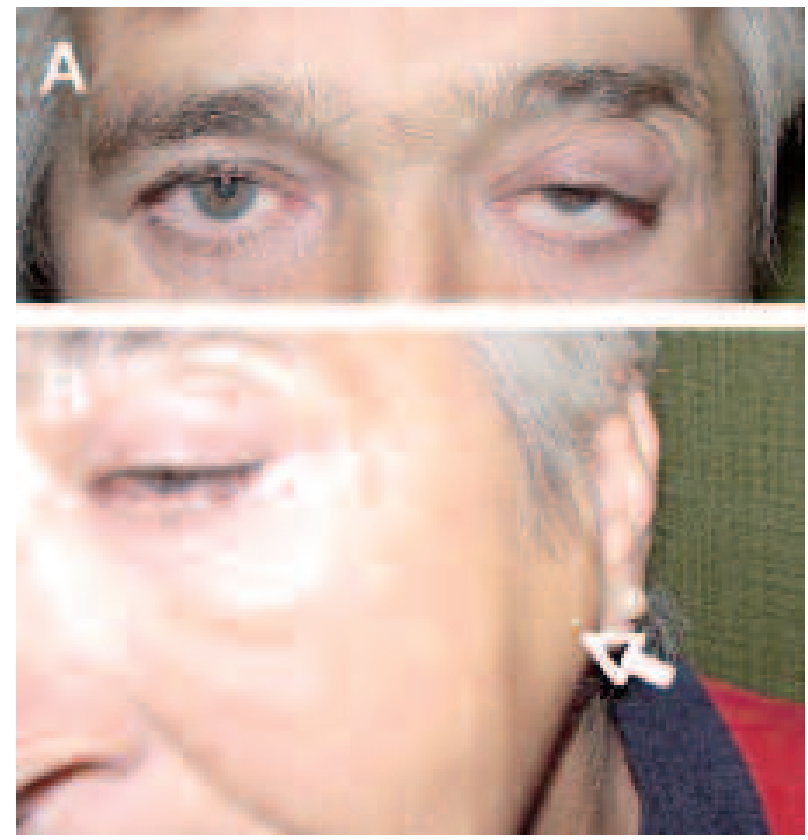

Fig. 6: Caso 6. La paciente presenta una ptosis palpebral izquierda causada por la tumoración en fosa lagrimal (A) y una tumoración preauricular izquierda (B).

objetivó una masa en el músculo recto medio del ojo derecho (fig. 7B), por lo que se remitió a nuestra Consulta de Oftalmología. Una PAAF no permitió el diagnóstico, por lo que se realizó una biopsia del tumor. Intraoperatoriamente se apreció una masa friable inmersa en el recto medio, compatible con linfoma. El diagnóstico anatomotológico de la biopsia fue de linfoma MALT, por lo que se instauró tratamiento quimioterápico con respuesta parcial. En la actualidad la paciente está estable.

\section{Caso 8}

Varón de 41 años de edad, sin antecedentes de interés, que en septiembre de 2004 fue atendido en nuestra Consulta de Oftalmología por presentar dolor en la hemimandíbula derecha irradiado hacia la órbita, con ptosis palpebral y exoftalmo de $4 \mathrm{~mm}$ en el ojo derecho (fig. 8A). Una RMN mostró una tumoración en el seno cavernoso derecho que producía un síndrome del apex orbitario (fig. 8B). Ante la imposibilidad de realizar una biopsia se inició un estudio sistémico en el que apareció una tumoración cecal que, mediante biopsia, se diagnosticó de linfoma B de células grandes, por lo que la masa en 

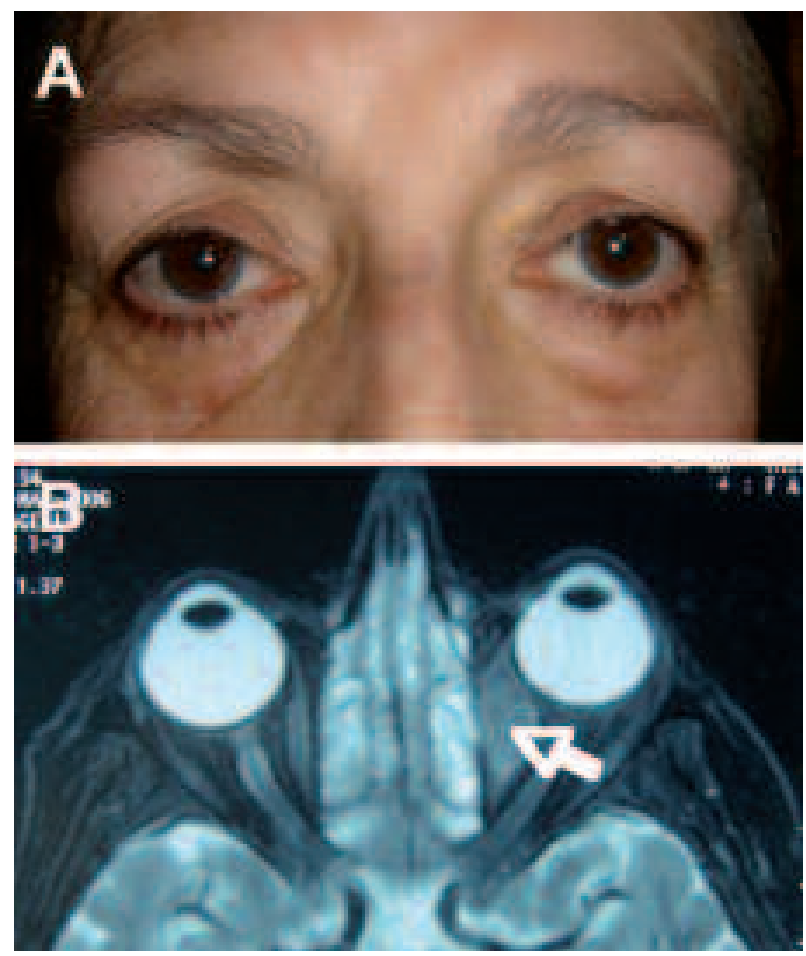

Fig. 7: Caso 7. La paciente (A) presenta una infiltración a nivel del recto medio del OI que se aprecia en la RMN (B) axial potenciada en $T 2$.

el seno cavernoso se supuso una metástasis de este tumor. El cuadro evolucionó desfavorablemente de forma rápida, apareciendo metástasis peritoneales, ascitis y derrame pleural, produciéndose el éxitus a las tres semanas de la primera visita.

\section{Caso 9}

Mujer de 78 años de edad que acudió a nuestra Consulta en agosto de 2006 por presentar exoftalmo y dolor en el ojo derecho de aproximadamente un año de evolución (fig. 9A). Una TAC orbitaria (fig. 9B) demostró la existencia de una masa sólida hiperdensa, captadora de contraste, que ocupaba el cuadrante ínfero-externo de la órbita derecha. Una PAAF realizada en septiembre de 2006 fue compatible con linfoma no Hodgkin, por lo que se remitió a hematología. Una biopsia de una adenopatía supraclavicular derecha y un estudio de extensión constató el diagnóstico definitivo de linfoma folicular grado II estadio IV. Se instauró tratamiento inmunoterápico y quimioterápico, con lo que remi-
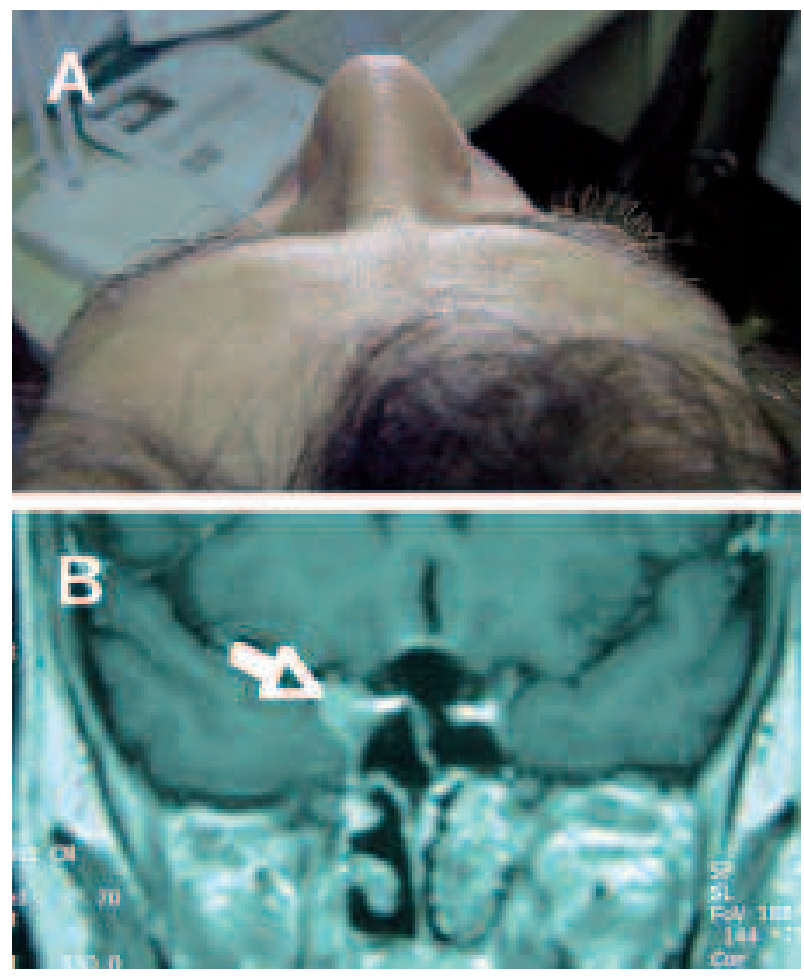

Fig. 8: Caso 8. La fotografía superior muestra un ligero exoftalmo derecho (A). En la RMN (B) cerebral coronal potenciada en $T 1$ con contraste intravenoso se aprecia una lesión a nivel del seno cavernoso (flecha) que alcanzaba el ápex de la órbita.

tió el cuadro. Actualmente la paciente se encuentra asíntomática y estable.

\section{DISCUSIÓN}

Los linfomas de la conjuntiva, glándula lagrimal, órbita y párpados, suelen ser tumores de bajo grado (2). Estudios recientes muestran que los linfomas suponen aproximadamente un $11 \%$ de todos los tumores de estas estructuras consideradas conjuntamente y un $55 \%$ de las tumoraciones malignas de esta zona (1,2). En nuestra casuística, los linfomas constituyen una de las tumoraciones orbitarias más frecuente, suponiendo aproximadamente un $15 \%$.

Las series publicadas muestran que la inmensa mayoría de los linfomas orbitarios (91\%) (2) debutan con clínica oftalmológica, consistente en masa conjuntival de color rosado o hiperemia conjuntival en el $32 \%$ de los casos, exoftalmo en el $27 \%$, masa palpebral u orbitaria en el $19 \%$, disminución de 

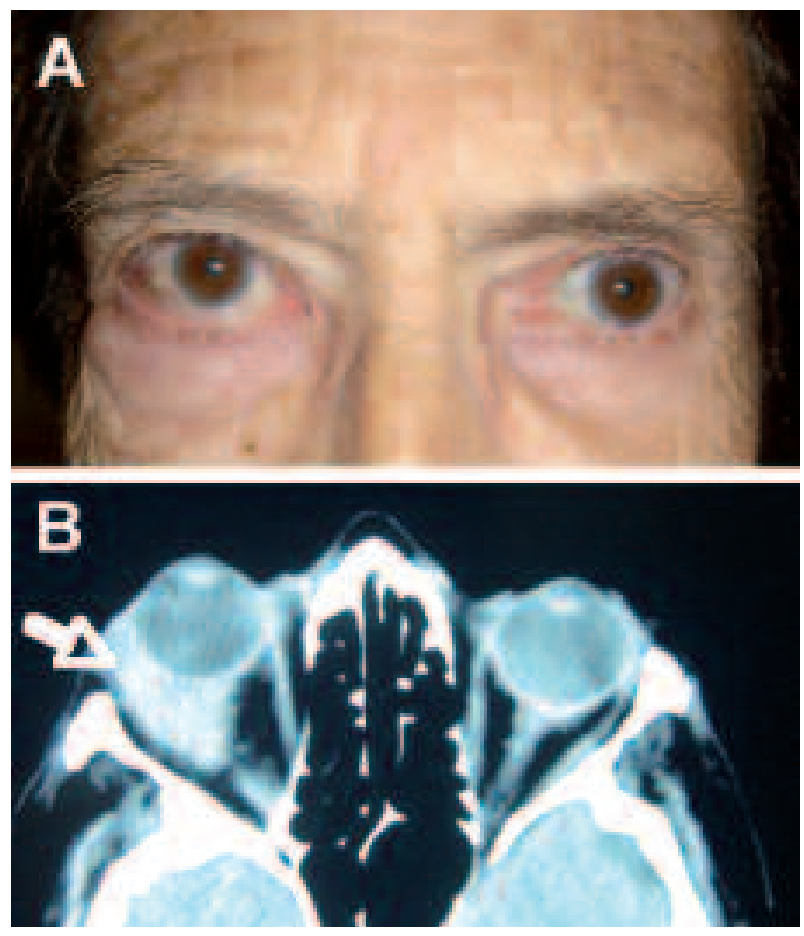

Fig. 9: Caso 9. En la figura superior (A) se observa el exoftalmo derecho de la paciente. La masa existente en el cuadrante infero-externo de la orbita derecha (flecha) se observa en la TAC axial con contraste intravenoso (B).

agudeza visual y ptosis en el $6 \%$, y diplopía en un $2 \%$ (2). En nuestra serie, la forma de presentación clínica más frecuente fue como masa palpebral u orbitaria (cuatro casos), seguida por exoftalmo (dos casos), ptosis palpebral (un caso), ptosis con exoftalmo (un caso) y ptosis con masa palpebral (un caso). A pesar de la frecuencia con que parece afectarse la conjuntiva ( $32 \%$ de casos) (2), nosotros no lo hemos observado en ninguno de nuestros pacientes.

El intervalo medio entre el inicio de los síntomas y la fecha del diagnóstico es de 4 a 6 meses (2-5). En nuestra serie hemos observado que hubo en la mayoría de los casos un intervalo de aproximadamente uno a dos meses. En un caso (caso 8), dada la evolución agresiva de la neoplasia, el diagnóstico se realizó aproximadamente a los 15 días del primer síntoma. En dos casos (casos 2 y 9), debido a la tardanza del paciente en acudir a consultar, el diagnóstico se hizo con un intervalo superior al año desde la aparición de la sintomatología. En uno de los casos (caso 7), desconocemos el tiempo transcurrido entre la aparición de la sintomatología y el diag- nóstico, puesto que el diagnóstico inicial de linfoma se había realizado seis años antes de que acudiese a nuestro Servicio de Oftalmología.

Las proliferaciones linfoides de los anejos oculares pueden ocurrir en todas las edades, pero aparecen más frecuentemente en la quinta o séptima década de la vida (2-9) y suelen afectar a 1,75 mujeres por cada hombre $(4-6,10)$. En nuestros casos la media de edad fue de 60 años en el momento del diagnóstico, siendo de 41 años el paciente más joven y de 78 el mayor. Estas edades son coincidentes con las publicadas en la literatura. En nuestra casuística hay cinco mujeres y cuatro varones, lo que indica una tendencia similar a la expuesta por estudios con series mayores.

Aproximadamente un $50 \%$ de los linfomas de anejos oculares se localizan la glándula lagrimal y otras áreas intraorbitarias, en especial la órbita antero-superior $(2,9,11,12)$. La conjuntiva se encuentra afectada en un tercio de los pacientes, y en el $96 \%$ de los casos consiste en un tumor de bajo grado (2). La afectación palpebral varía según las distintas series de casos entre el $0 \%$ y el $44 \%$, siendo la media de los distintos trabajos publicados en torno al $10 \%$ (2). Aproximadamente del 10 al 17\% de los casos el linfoma se presenta bilateralmente (3-5), apareciendo de forma simultánea en ambas órbitas aproximadamente el $80 \%$ de los casos bilaterales, y subsecuentemente en el $20 \%$ de las ocasiones (13). Otras publicaciones amplían la horquilla del porcentaje de bilateralidad situándola entre el 7 al 24\% de los casos (2,9). Entre un 20 y un $40 \%$ de los pacientes tienen historia de linfoma extraorbitario en el momento de diagnosticar un linfoma orbitario $(4,5,14)$. La enfermedad difusa linfomatosa se encuentra principalmente en pacientes con linfoma no Hodgkin de alto grado. El $26 \%$ de pacientes con linfoma de bajo grado alcanzan el estadio IV, mientras que lo hacen el $56 \%$ de pacientes con linfomas de alto grado $(2,9)$.

En nuestro estudio, el linfoma se localizó intraorbitario en tres casos (en las zonas ínferoexterna, inferior y medial), en la fosa lagrimal en un caso, compartiendo párpado y parte de la órbita en tres casos, sólamente en el párpado en un caso y en el seno cavernoso en un caso. No hemos encontrado afectación conjuntival ni bilateralidad en ningún caso. Hubo linfoma con localización extraorbitaria en el momento del diagnóstico en siete de los nueve casos. En un caso (caso 6) se encontró enfermedad extraorbitaria en el seguimiento posterior, pues 
la paciente acudió tardíamente a las visitas para completar el estadiaje y realizar el tratamiento. Aunque disponemos de pocos casos para considerarlo significativo, nuestra serie presenta una concurrencia de linfoma extraorbitario mayor de lo esperado.

Coupland y colaboradores (4) encontraron en su casuística de 112 pacientes con linfomas de anejos oculares, un $64 \%$ de linfomas de células B de la zona marginal extranodales (MALT), un $10 \%$ de linfomas centro foliculares, un $9 \%$ de linfoma de células B difusas grandes, un 6\% de plasmocitomas, y un 5\% de linfoma linfoplasmocítico. Por otra parte, McKelvie y colaboradores (15) en una serie de 73 casos de linfomas de anejos oculares hallaron un $63 \%$ de linfomas MALT, un $17 \%$ de linfomas foliculares, un $11 \%$ de linfomas de célula B difusas grandes, un $3 \%$ de linfomas de células del manto, un $3 \%$ de leucemia linfocitica crónica de células B/linfoma linfocítico pequeño, un $1,5 \%$ de linfoma de células $\mathrm{T}$ periféricas, y un 1,5\% de linfomas de células Natural Killer. Prácticamente todas los estudios coinciden en que el tipo más común de linfoma no Hodgkiniano de órbita y anejos oculares es el linfoma MALT $(4,15,16)$. En nuestra casuística encontramos tres linfomas MALT, un linfoma B de bajo grado, dos linfomas no Hodgkin B de células grandes, dos linfomas foliculares y un linfoma del manto, lo cual se corresponde con otros estudios encontrados en la literatura.

Publicaciones recientes hacen referencia a la posible asociación de linfomas MALT con la infección por Chlamydia Psittachi $(2,16,17)$. Esto hace, que aunque la radioterapia haya sido el tratamiento estándar para los linfomas oftalmológicos localizados, están apareciendo otras opciones de posible valor terapéutico como la terapia antibiótica para Chlamydia Psittaci. También la terapia con anticuerpos monoclonales CD20 puede constituir una alternativa prometedora a la irradiación externa y su potencial toxicidad (2).

En términos generales los hallazgos obtenidos en nuestra serie son muy similares a los hallados en la literatura. Es importante que el oftalmólogo conozca este tipo de patología orbitaria, pues en muchas ocasiones, es el primero en contactar con el paciente que la padece. Su detección precoz es muy importante, ya que se trata de una enfermedad potencialmente curable. Especialmente relevante resulta la realización de una minuciosa historia clí- nica, puesto que no es infrecuente la presencia de afectación extraorbitaria en el momento en el que aparece clínica oftalmológica. En estudios recientes se ha observado un incremento de la incidencia de linfomas no Hodgkin orbitarios, por lo que es importante conocer esta entidad para evitar que pase inadvertida (18).

\section{BIBLIOGRAFÍA}

1. Shields JA, Shields CL, Scartozzi R. Survey of 1264 patients with orbital tumors and simulating lesions: The 2002 Montgomery Lecture, part 1. Ophthalmology 2004; 111: 997-1008.

2. Decaudin D, de Cremoux P, Vicent-Salomon A, Dendale $R$, Rouic LL. Ocular adnexal lymphoma: a review of clinicopathologic features and treatment options. Blood 2006; 108: 1451-1460.

3. Coupland SE, Hummel M, Stein H. Ocular adnexal lymphomas: five case presentations and a review of the literature. Surv Ophthalmol 2002; 47: 470-490.

4. Coupland SE, Krause L, Delecluse HJ, Anagnostopoulos I, Foss HD, Hummel M, et al. Lymphoproliferative lesions of the ocular adnexa. Analysis of 112 cases. Ophthalmology 1998; 105: 1430-1441.

5. Knowles DM, Jacobiec FA, McNally L, Burke JS. Lymphoid hyperplasia and malignant lymphoma ocurring in the ocular adnexa (orbit, conjunctiva, and eyelids): a prospective multiparametric analysis of 108 cases during 1977 to 1987. Hum Pathol 1990; 21: 959-973.

6. Auw-Haedrich C, Coupland SE, Kapp A, Schmitt-Gräff A, Buchen R, Witschel H. Long term outcome of ocular adnexal lymphoma subtyped according to the REAL classification. Revised European and American lymphoma. Br J Ophthalmol 2001; 85: 63-69.

7. Jenkins C, Rose GE, Bunce C, Wright JE, Cree IA , Plowman $N$, et al. Histological features of ocular adnexal lymphoma (REAL classification) and their association with patient morbility and survival. Br J Ophthalmol 2000; 84: 907-913.

8. Shields CL, Shields JA, Carvalho C, Rundle P, Smith AF. Conjuntival lymphoid tumors: clinical analysis of 117 cases and relationship to systemic lymphoma. Ophthalmology 2001; 108: 979-984.

9. Meunier J, Lumbroso-Le Rouic L, Vicent-Salomon A, Dendale R, Asselain B, Arnaud P, et al. Ophthalmologic and intraocular non-Hodgkin's lymphoma: a large single center study of inicial characteristics, natural history, and pronostic factors. Hematol Oncol 2004; 22: 143-158.

10. White WL, Ferry JA, Harris NL, Grove AS Jr. Ocular adnexal lymphoma. A clinicopathologic study with identification of lymphomas of mucosa-associated lymphoid tissue type. Ophthalmology 1995; 102: 1994-2006.

11. Martinet S, Ozsahin M, Belkacémi Y, Landmann C, Poormans $P$, Oehlere $C$, et al. Outcome and prognostic factors in orbital lymphoma: a Rare Cancer Network study on 90 consecutive patients treated with radiotherapy. Int $J$ Radiat Oncol Biol Phys 2003; 55: 892-898. 
12. Uno T, Isobe $K$, Shikama N, Nishikawa A, Oguchi M, Ueno $N$, et al. Radiotherapy for extranodal, marginalzone, B-cell lymphoma of mucosa-associated lymphoid tissue originating in the ocular adnexa: a multiinstitutional, retrospective review of 50 patients. Cancer 2003; 98: 865871.

13. McNally L, Jakobiec FA, Knowles DM. Clinical, morphologic, immunophenotypic, and molecular genetic analysis of bilateral ocular adnexal lymphoid neoplasms in 17 patients. Am J Ophthalmol 1987; 103: 555-568.

14. Johnson TE, Tse DT, Byrne GE Jr, Restrepo A, Whitcomb CC, Voigt W, et al. Ocular-adnexal lymphoid tumors: a clinicopathologic and molecular genetic study of 77 patients. Ophthal Plast Reconstr Surg 1999; 15: 171-179.
15. McKelvie PA, McNab A, Francis IC, Fox R, O'Day J. Ocular adnexal lymphoproliferative disease: a series of 73 cases. Clin Experiment Ophthalmol 2001; 29: 387-393.

16. Ferreri AJ, Ponzoni M, Guidoboni M, Resti AG, Politi LS, Cortelazzo S, et al. Bacteria-eradicating therapy with doxycycline in ocular adnexal MALT lymphoma: a multicenter prospective trial. J Natl Cancer Inst 2006; 98: 1375-1382.

17. Shen D, Yuen HK, Galita DA, Chan NR, Chan CC. Detection of Chlamydia pneumoniae in a bilateral orbital mисоsa-associated lymphoid tissue lymphoma. Am J Ophthalmol 2006; 141: 1162-1163.

18. Moslehi R, Devesa SS, Schairer C, Fraumeni JF Jr. Rapidly increasing incidence of ocular non-hodgkin lymphoma. J Natl Cancer Inst 2006; 98: 936-939. 